\title{
Association of Bovine Leukocyte Antigen (BoLA) DRB3.2 with Immune Response, Mastitis, and Production and Type Traits in Canadian Holsteins
}

\author{
R. Rupp, ${ }^{*} \dagger$ A. Hernandez, ${ }^{*}$ and B. A. Mallard ${ }^{\star 1}$ \\ *Department of Pathobiology, Ontario Veterinary College, University of Guelph, Guelph, Ontario, Canada N1G 2W1 \\ †Station d'Amélioration Génétique des Animaux, Institut National de la Recherche Agronomique, 31326 Castanet-Tolosan, France
}

\begin{abstract}
Data collected from 328 Canadian Holsteins in a research herd at the University of Guelph were used to study associations among expression of bovine leukocyte antigen (BoLA) DRB3.2 alleles, immune response, mastitis resistance via somatic cell counts (SCC), and clinical mastitis, as well as to extend these results to production and type traits. Accordingly, groups of cows were evaluated in vivo for both the antibody-mediated immune response (AMIR) and the cell-mediated immune response (CMIR), which generally predominate in responses to extracellular and intracellular pathogens, respectively. Of note was that associations between BoLA DRB3.2 alleles and immune responses tended to be in the opposite sign for the 2 AMIR and CMIR traits examined. For example, alleles DRB3. $2 * 3$ and $* 24$ were associated with higher AMIR but lower CMIR, whereas allele $* 22$ was associated with lower AMIR but higher CMIR. This finding is in agreement with the hypothesis that both traits are genetically independent and represent opposing type 1 and type 2 immune responses. Additionally, BoLA DRB3.2*3 and *11 were associated with lower SCC, whereas alleles $* 22$ and $* 23$ were associated with higher SCC. Finally, allele DRB3.2*3 was also associated with less clinical mastitis, whereas allele $* 8$ was associated with higher mastitis risk. Allele $* 3$ was of particular relevance because it was associated with increased antibodies, as well as reduced mastitis and SCC. This could be due to an indirect relationship between the ability to produce a high antibody response and enhanced defense against intrammamary infections caused by extracellular pathogens. Consequently, the BoLA DRB3.2*3 allele should be investigated further as a candidate for resistance to some types of intramammary infections, the important caveat being its association with lower CMIR, particularly with one of the test antigens used
\end{abstract}

Received April 26, 2006.

Accepted August 27, 2006.

${ }^{1}$ Corresponding author: bmallard@uoguelph.ca to evaluate delayed-type hypersensitivity. The results of associations between BoLA DRB3.2 and production traits were, in some cases, antagonistic in that BoLA DRB3.2 alleles $* 11$ and $* 23$, which are associated with increased production traits, were associated with lower and higher SCC, respectively. Collectively, these findings advocate the use of alleles $* 3, * 23$, and $* 22$ as reference points for more detailed mechanistic studies. This does not imply that genetic selection for mastitis resistance should be based on BoLA alleles, but that information on a variety of genes may aid in identification and selection for improved health.

Key words: immune response, mastitis, bovine leukocyte antigen, dairy cattle

\section{INTRODUCTION}

Improving the health of livestock is internationally of increasing importance to the dairy industry and to consumers (Stear et al., 2001). Other than economic gains that can be expected from reduced production costs (reducing drug costs, production losses, and the replacement rate), concern emerging in the 1990s also relates to increased food safety, reduced use of drugs (including antibiotics), and animal welfare. In ruminants, polygenic variation of resistance to disease is well documented, and many countries have recently updated their breeding objectives to include or increase the weight for nonproductivity traits, with special attention given to mastitis resistance (Rupp and Boichard, 2003). However, the genes and mechanisms involved in disease resistance in ruminants are mostly unknown, efficacious vaccines against IMI are not available, and research to enhance animal defenses is ongoing. Therefore, investigation of the role of possible major genes, such as genes encoding the major histocompatibility complex (MHC) remains relevant.

Indeed, the MHC plays an essential role in the induction and regulation of acquired immune response and has been associated with resistance to a variety of diseases (Rothschild et al., 2000). Genes encoding the MHC are highly polymorphic, and numerous associations between allelic variants and immune respon- 
siveness and disease resistance have been reported (Stear et al., 2001). In some cases breeding programs based on MHC associations have been successfully implemented to alter disease occurrence (Maillard et al., $2002,2003)$. In dairy ruminants, nearly all of the most recent studies have focused on the exon 2 of the Class II DRB3 locus because of its high polymorphism (http:// www.projects.roslin.ac.uk/bola/) and because it encodes the antigen-binding site of MHC molecules (Rothschild et al., 2000). Several studies have reported a relationship between bovine MHC (BoLA, bovine leukocyte antigen) class I molecules and resistance or susceptibility to mastitis (Weigel et al., 1990; Mejdell et al., 1994; Aarestrup et al., 1995; Mallard et al., 1995) or immune response (Mallard et al., 1995). Others have reported a relationship between class II DRB variants and resistance or susceptibility to mastitis (Dietz et al., 1997; Kelm et al., 1997; Starkenburg et al., 1997; Sharif et al., 1998a), with replication of bovine leukemia virus in cattle (Xu et al., 1993) and sheep (Konnai et al., 2003 ), and with protection induced by peptide vaccines against foot-and-mouth disease in cattle (Garcia-Briones et al., 2000). Maillard et al. (2003) identified a DR/ DQ haplotype that was highly correlated with resistance or susceptibility to dermatophilosis in cattle, and Raadsma et al. (1999) found 4 ovine MHC haplotypes to be associated with vaccine response to Dichelobacter nodosus in sheep. Nonetheless, there are still conflicting results regarding associations between the same the BoLA alleles and phenotypes across studies, and a clearly contrasting association of some BoLA alleles with resistance to different diseases or with immune responses (Xu et al., 1993; Sharif et al., 1998a). Therefore, additional studies are still required to confirm associations within and between populations. Moreover, investigating the relationships between BoLA variants and host defense mechanisms is desirable to better understand underlying biological mechanisms and potentially to use the results to improve genetic or vaccination strategies for increased disease resistance in livestock.

The aim of this study was to reassess the previous results of associations between BoLA DRB3.2 alleles and mastitis resistance (via SCC) in Canadian dairy cattle, and to extend the investigation to production and type traits. Furthermore, relationships were explored between BoLA DRB3.2 variants and immune response components. Accordingly, cows were evaluated in vivo for both antibody-mediated immune response (AMIR), which often predominate against extracellular pathogens, and cell-mediated immune response (CMIR), which involve T-leukocytes as effectors and largely aid in protection against intracellular pathogens.

\section{MATERIALS AND METHODS}

\section{Animals}

Data were collected from 1990 through 2005 from Holstein cows housed in the research herd of Ontario Veterinary College (University of Guelph, Guelph, Ontario, Canada). Animals were raised in 2 locations, the Elora and Ponsonby barns. A total of 328 cows having the BoLA genotype, SCC, and milk production records in first lactation were used. Most of the primiparous cows also had information for fat and protein yields and for type traits. Later lactation records for SCC and production traits for the multiparous cows were also considered, resulting in a total data set of 831 lactations, including 328, 248, and 167 first- to third-lactation records. Cell-mediated immune response and AMIR were both evaluated at the same time and on the same animals. Because immune response recording was laborious and time-consuming, measures were done on successive groups of cows in 2002 ( 1 group of 20 cows) and 2004 (3 groups of 32, 31, and 18 cows between July and December). Out of 101 animals measured, 90 and 81 valid CMIR and AMIR data, respectively, were available.

Clinical mastitis, diagnosed by trained experimental farm technicians, was recorded for 128 BoLA-genotyped animals, including 83 cows with immune response and all their contemporaries with health records in the first 3 lactations between 2003 and 2005. In total, mastitis data from 128, 117, and 49 first, second, and third lactations, respectively, were available. The 328 cows in the complete data set were born from 114 sires, with 6 of these sires having between 10 and 24 daughters.

\section{Genotyping of BoLA-DRB3}

Extraction of DNA from blood mononuclear cells was done as described elsewhere (Sharif et al., 1998a). The second exon of the BoLA DRB3 gene was amplified and genotyped according to the PCR-RFLP method described by van Eijk et al. (1992). Minor modifications were added. For the first round of PCR, $4 \mu \mathrm{L}$ of cell lysate was added to $16 \mu \mathrm{L}$ of PCR mixture (final concentration: $0.25 \mathrm{p} M$ of primers, $0.2 \mathrm{~m} M$ of dNTP, $1.8 \mathrm{p} M$ of $\mathrm{MgCl}_{2}$, and 1 unit of Taq DNA polymerase; Invitrogen, Carlsbad, CA). Subsequently, $6 \mu \mathrm{L}$ of first-round product was added to another $24 \mu \mathrm{L}$ of PCR mixture (final concentration: $0.80 \mathrm{p} M$ of primers, $0.25 \mathrm{~m} M$ of dNTP, $1.5 \mathrm{p} M$ of $\mathrm{MgCl}_{2}$, and 1 unit of Taq DNA polymerase; Invitrogen) for a second round of PCR following van Eijk et al. (1992). Products of PCR were digested with RsaI, BstYI, and HaeIII restriction enzymes. Digestion reactions were incubated overnight and digestion products were resolved on an $8 \%$ polyacrylamide gel using 
a MiniProtean apparatus (Mini-Protean II electrophoresis cell; Bio-Rad, Hercules, CA) by running at $80 \mathrm{~V}$ for $65 \mathrm{~min}$. The BoLA genotypes were determined according to the BoLA allelic nomenclature described by van Eijk et al. (1992) and reported on the BoLA nomenclature homepage (http://www.projects.roslin.ac.uk/ bola/drb3pcr.html\#table).

\section{AMIR and CMIR}

As described previously (Wagter et al., 2000; Hernandez et al., 2003), ovalbumin (OVA) and hen egg white lysozyme (HEWL) were used as a test antigens, and secondary AMIR was measured by ELISA (reported as optical density) on d 21 following immunizations on d 0 and 14. Two test antigens known to induce delayedtype hypersensitivity (DTH) were used as an in vivo measurement of CMIR (Mallard et al., 1998; Hernández et al., 2003, 2005). Measurements ( $\mathrm{mm}$ ) of the increase in double skinfold thickness at 24 and $48 \mathrm{~h}$ postelicitation of DTH, as well as the average DTH response (24 $+48 \mathrm{~h}$ ), were taken using calipers (Harpenden skinfold caliper; Creative Health Products, Inc., Ann Arbor, MI). Phosphate buffered saline was used as the negative control.

\section{SCC, Clinical Mastitis, and Production and Type Traits}

Test-day SCC; 305-d milk, fat, and protein; and type trait classifications were extracted from the Canadian Dairy Network database (Guelph, Ontario, Canada), along with birth dates, calving dates, factors affecting type trait records (classifier, round, classification date), and pedigree information. Test-day SCC were classically transformed to SCS $\left[\mathrm{SCS}=\log _{2}(\mathrm{SCC} / 100)+3\right.$ (Ali and Shook, 1980)] to achieve normality of data distribution. The trait considered in this study was the arithmetic mean of test-day SCS (LSCS) for a given lactation with at least 3 test-day SCS records.

The clinical mastitis trait was defined as the presence or absence (binary trait) of at least one case of mastitis during a given lactation. Repeated binary measures per cow were available for the first 3 lactations.

Production traits were the standard 305-d milk, fat, and protein yields. Type traits of interest were those that are currently included in the Lifetime Profitability Index for Canadian dairy cows (Canadian Dairy Network homepage, http://www.cdn.ca/Articles/0502/lpiFeb2005.pdf) and that are potentially related to disease occurrence, such as mastitis and longevity. Those traits were udder depth (UD), capacity (CAP), mammary system (MS), and feet and legs (FL). These traits were evaluated once during the first lactation.

\section{Statistical Analyses}

Method. Gene-substitution, linear, mixed models were used to evaluate the effect of BoLA DRB3.2 alleles on the different continuous traits: immune responses, LSCS, and production and type traits (PROC MIXED; SAS Institute, Cary, NC). Logistic regression was applied to the gene substitution models for repeated binary measures of clinical mastitis (PROC GENMOD, repeated option; SAS Institute). Therefore, the binary nature of udder health data was taken into account, as was within-cow correlation for repeated mastitis occurrence across lactations.

The following procedure was applied to define the models. In a first step, classical environmental effects and a sire effect were tested for each trait without considering the BoLA DRB3.2 effect. Sires were relatively well distributed over the data set, with a large number of sires with few daughters. Several AI sires, however, had a large number of daughters, which can potentially lead to confusion between a BoLA DRB3.2 allele effect and a sire effect. Therefore, a fixed sire effect was defined with 7 levels: one level for each of the 6 mostused sires, and one level for all others. Including sire effect as a random effect was also tested. Significant ( $P$ $\leq 0.10$ ) effects were subsequently applied in full models, including the vector of BoLA allele effects. Similar parameterization of the BoLA DRB3.2 allele effect was applied to all traits, following Sharif et al. (1998a), to facilitate comparison across traits and comparison with results among these studies. The BoLA DRB3.2 allele $* 24$ was therefore set to zero, whereas alleles $* 3, * 8$, $* 11, * 16, * 22, * 23$, and an "other" category (pooling the other alleles with low frequencies) were included in the model. Results were expressed as differences from the *24 allele.

Models for Immune Response Traits. Models for the immune response traits were as follows:

$$
\mathrm{Y}_{\mathrm{ij}}=\mathrm{X}_{\mathrm{i}} \beta_{\mathrm{i}}+\Sigma_{\mathrm{j}} \alpha_{\mathrm{j}} \mathrm{BoLA}_{\mathrm{j}}+\mathrm{e}_{\mathrm{ij}}
$$

where $\mathrm{Y}_{\mathrm{ij}}$ is $\mathrm{AMIR}_{\mathrm{d} 21}, \mathrm{CMIR}_{24 \mathrm{~h}}, \mathrm{CMIR}_{48 \mathrm{~h}}$, or $\mathrm{CMIR}_{\text {average; }}$; BoLA $_{j}$ is the fixed effect of number of copies $(0,1$, or 2$)$ of BoLA allele $\mathrm{j}(\mathrm{j}=* 3, * 8, * 11, * 16, * 22, * 23$, others); $\alpha_{\mathrm{j}}$ is the regression coefficient on the number of copies of the jth BoLA allele; $\Sigma_{\mathrm{j}}$ is the summation from 1 to $\mathrm{j} ; \mathrm{X}_{\mathrm{i}}$ is the vector of environmental effects; $\beta_{\mathrm{i}}$ are the regression coefficients on the vector of fixed effects $\mathrm{X}$; and $\mathrm{e}_{\mathrm{ij}}$ is the random error term. The environmental fixed effects tested were parity, calving year (2001 to 2004), calving season (spring, summer, fall, and winter), experimental group of the cow (1 in 2002, and 2, 3 , and 4 in 2004) and stage at the time of measurement (DIM grouped into 4 classes). Because experimental 
group and herd were confounded, the herd effect was not tested. Effects included in $\mathrm{X}(P \leq 0.10)$ were calving season and lactation stage for CMIR and none for AMIR, given that these effects were not significant in preliminary analyses.

Models for LSCS and Production Traits. Models for LSCS and production traits were as follows:

$$
\mathrm{Y}_{\mathrm{ijk}}=\mathrm{X}_{\mathrm{i}} \beta_{\mathrm{i}}+\Sigma_{\mathrm{j}} \alpha_{\mathrm{j}} \mathrm{BoLA}_{\mathrm{j}}+\mathrm{a}_{\mathrm{k}}+\mathrm{e}_{\mathrm{ijk}},
$$

where $Y_{\mathrm{ijk}}$ is LSCS, milk, fat, or protein in the first 3 lactations; $B_{0 L A}$ is the fixed effect of number of copies $(0,1$, or 2$)$ of BoLA allele $\mathrm{j}(\mathrm{j}=* 3, * 8, * 11, * 16, * 22, * 23$, others); $\alpha_{\mathrm{j}}$ is the regression coefficient on the number of copies of the jth BoLA allele; $\Sigma_{\mathrm{j}}$ is the summation from 1 to $\mathrm{j} ; \mathrm{X}_{\mathrm{i}}$ is the vector of environmental effects; $\beta_{\mathrm{i}}$ are the regression coefficients on the vector of fixed effects $\mathrm{X} ; \mathrm{a}_{\mathrm{k}}$ is the random animal effect; and $\mathrm{e}_{\mathrm{ijk}}$ is the random error term.

The environmental fixed effects tested were parity ( 1 to 3), calving year (1990 to 2004), calving month (January to December), age at calving within parity (24 to $29 \mathrm{mo}, 36$ to $44 \mathrm{mo}, 48$ to $55 \mathrm{mo}$ in lactation 1, 2, and 3, respectively), and herd (Elora or Ponsonby). The model for LSCS and production traits also included a random animal effect, because cows had repeated measures across lactations. Fixed effects included in X $(P \leq 0.10)$ were parity; calving year within parity; and calving month within parity, herd, and sire. For LSCS, analyses also were performed separately for each lactation (without the random animal effect), as well as on the subset of 114 cows with AMIR or CMIR information.

Models for Clinical Mastitis. The logistic regression model for clinical mastitis was as follows:

$$
\mathrm{Y}_{\mathrm{ij}}=\mathrm{X}_{\mathrm{ij}} \beta_{\mathrm{ij}}+\Sigma_{\mathrm{k}} \alpha_{\mathrm{k}} \text { BoLA }_{\mathrm{k}}+\mathrm{e}_{\mathrm{ij}},
$$

where $\mathrm{Y}_{\mathrm{ij}}$ is the $\log (P / 1-P)$, where $P$ is the probability that cow $\mathrm{i}$ is affected by at least one case of clinical mastitis during parity $\mathrm{j}$; $\mathrm{BoLA}_{\mathrm{k}}$ is the fixed effect of number of copies $(0,1$, or 2$)$ of BoLA allele $\mathrm{k}(\mathrm{k}=* 3$, $* 8, * 11, * 16, * 22, * 23$, others); $\alpha_{\mathrm{k}}$ is the regression coefficient on the number of copies of the jth BoLA allele; $\Sigma_{\mathrm{j}}$ is the summation from 1 to $\mathrm{j} ; \mathrm{X}_{\mathrm{ij}}$ is the vector of environmental effects; $\beta_{\mathrm{ij}}$ are regression coefficients on the vector of fixed effects $\mathrm{X}$; and $\mathrm{e}_{\mathrm{ij}}$ is the random error term.

Effects included in $\mathrm{X}(P \leq 0.10)$ were calving season, calving year, parity, herd, and sire. An unstructured correlation matrix for responses was fitted to model the correlation of the responses within cows.

Models for Type Traits. Models for the 4 type traits (UD, MS, CAP, or FL) were similar to models for other traits reported above. The fixed effects included $(P \leq$
0.10) were classifier (13 classes) for all 4 type traits; lactation stage at classification (9 classes) for FL, MAM, and CAP; round (11 classes); calving season (spring, summer, fall, and winter) and calving year (1991 to 2004) for MAM and UD; age at classification (25 to 34 mo) for FL and MAM; and sire for FL and UD.

\section{RESULTS}

\section{BoLA DRB3.2 Genotyping and Basic Statistics}

The distribution of BoLA DRB3.2 allele frequencies is shown in Figure 1. The most frequent alleles were BoLA DRB3.2*8, *22, *24, and *16, with frequencies ranging from 12 to $18 \%$. The alleles BoLA DRB3. $2 * 11$, $* 3$, and $* 23$ were the second most represented group of alleles, with frequencies between 6 and 9\%. Ranking of these alleles was largely conserved in the subset of data that included only cows evaluated for immune response (Figure 1) and over the time period of year at first calving (Figure 2). Allele frequencies were relatively stable in the research herd in the last $10 \mathrm{yr}$, although BolA DRB3.2*24 and *8 were more frequent $(\geq 20 \%)$ in the early $1990 \mathrm{~s}$, whereas the frequency of BoLA DRB3.2*3, a seemingly favorable allele, increased regularly from 1996 to 2004 .

Mean AMIR and CMIR values are given in Table 1. The average CMIR response to both test antigens was similar (89 and 94\% increase in skinfold thickness). We observed the CMIR response to be slightly higher at 24 $\mathrm{h}$ (100\% increase) than at $48 \mathrm{~h}$ (78\% increase) for Ag1 , whereas no difference was observed between 24 - and 48-h responses for Ag-2. The immune response was generally measured during one of the first 3 lactations: $48 \%$ in the first lactation, $34 \%$ in the second lactation, and $18 \%$ in third or later lactations. However, parity had no significant effect $(P \leq 0.10)$ on AMIR or CMIR traits in this data set and was not included in the mixedmodel analyses. The distribution of both AMIR and CMIR data was close to normal, so no transformation of raw data was required.

The mean LSCS of all cows (Table 1) was comparable to the LSCS (SD) for the subset of cows that were evaluated for immune response ( $\mathrm{n}=114$, data not shown). In total, 118 cases of clinical mastitis were diagnosed in 294 lactations of 128 cows. The frequency of clinical mastitis was $20.3 \%$ in primiparous cows and slightly higher in older cows (Table 1). In $60 \%$ of affected cows, only one case of mastitis occurred, whereas 2 or more cases per lactation were recorded in only 26 and $17 \%$ of affected cows, respectively.

\section{Association Between BoLA DRB3.2 Alleles and Immune Response}

The BoLA DRB3.2 allele substitution effects (estimates from the mixed models) on CMIR and AMIR are 


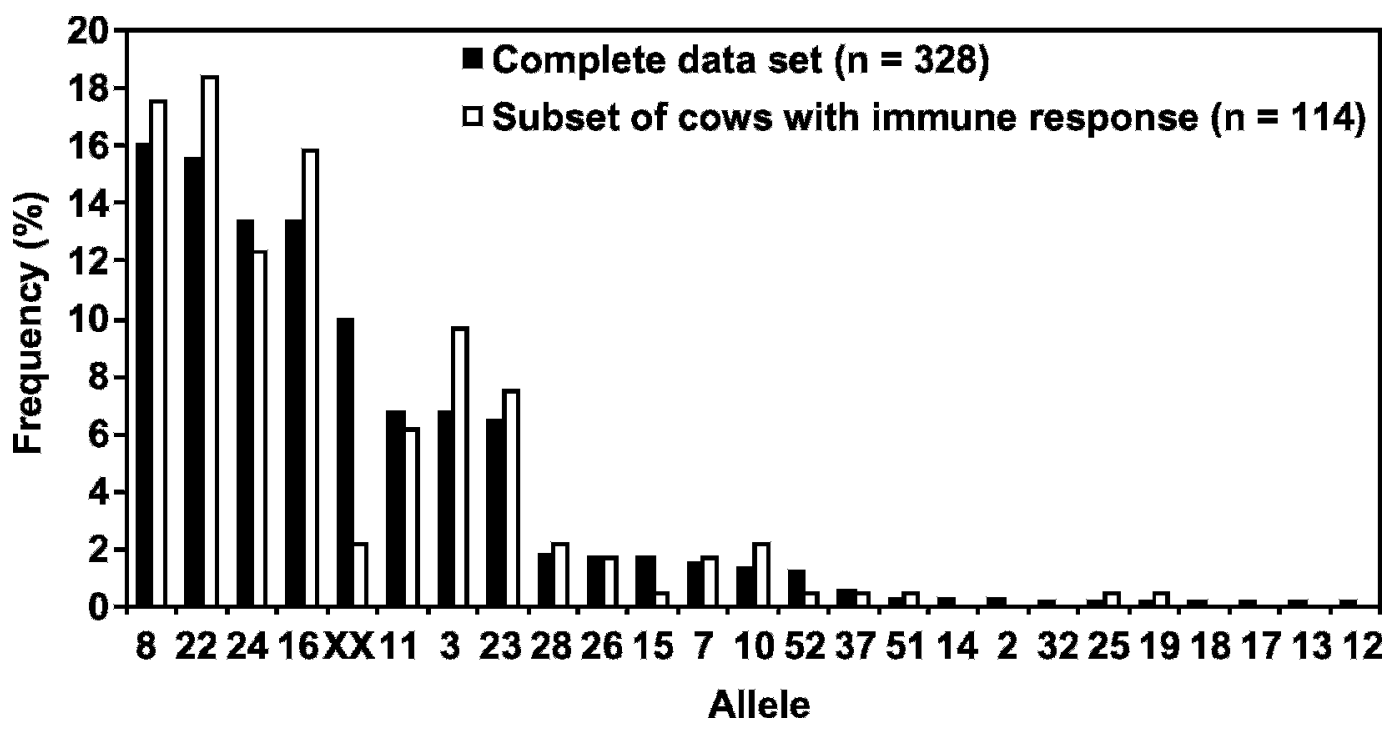

Figure 1. Distribution of bovine leukocyte antigen (BoLA) DRB3.2 allele frequencies in the University of Guelph research herds (XX = undetermined allele).

shown in Figures 3 and 4. For a given test antigen, results for the CMIR response were very similar, whether 24- or 48-h responses were considered. Therefore, only results based on the average CMIR response (means of 24- and 48-h DTH responses) are shown. Polymorphism at the BoLA did not significantly influence DTH to Ag-1. No BoLA DRB3.2 allele was significantly $(P \leq 0.05)$ associated with a higher or lower CMIR response to test $\mathrm{Ag}-2$, when compared with the reference DRB3.2*24 effect set to zero (Figure 3). However, the contrast between the allele $* 22$ and allele $* 3$ effect (data not shown) was significant $(P=0.03)$. Therefore,

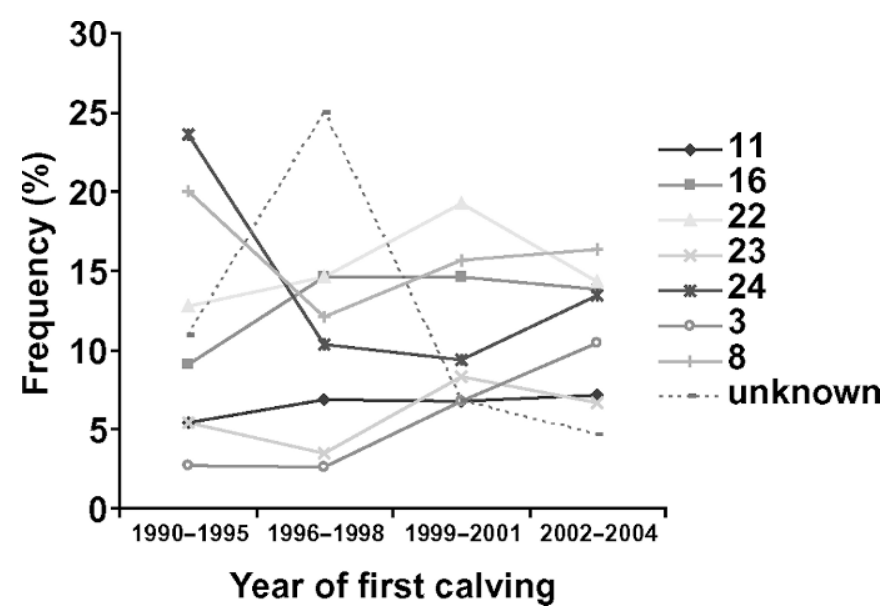

Figure 2. Distribution of bovine leukocyte antigen (BoLA) DRB3.2 allele frequencies according to time period (year of first calving) in the University of Guelph research herds.
DRB3.2*22 was found to be associated with a greater DTH response, whereas DRB3.2*3 was associated with lower DTH. Furthermore, DRB3.2*22 tended to be associated with higher DTH responses $(P=0.07)$ than DRB3.2*24, and this contrast was significant $(P=0.03)$ when the 48-h DTH response to test Ag-2 was considered (data not shown).

The association between BoLA DRB3.2 alleles and the AMIR response to OVA or HEWL showed opposite results (Figure 4). Bovine leukocyte antigen DRB3.2*22 was significantly $(P \leq 0.001)$ associated with less antibody than DRB3.2*24. Furthermore, contrasts of the DRB3.2*22 effect were significant $(P \leq 0.05)$ when compared with each of the other 5 alleles $(* 3, * 8, * 11, * 16$, $* 23$; data not shown). Therefore, allele *22 was clearly associated with lower AMIR, whereas cows expressing DRB3. $2 * 3, * 16$, and $* 24$ had the highest antibody response at $21 \mathrm{~d}$ postimmunization $(P \leq 0.0005)$.

\section{Association Between BoLA DRB3.2 Alleles and LSCS}

The association between BoLA DRB3.2 alleles and LSCS in the complete data set $(\mathrm{n}=328$ cows and 821 lactations) is reported in Table 2 . Bovine leukocyte antigen DRB3.2*11 was significantly $(P=0.04)$ associated with lower cell counts when compared with the reference allele BoLA DRB3.2*24. Other significant contrasts (Table 2 ) were between allele $* 23$ and allele $* 11$ (+0.68; $P=0.002)$, and between allele $* 23$ and allele $* 3$ (+0.52, $P=0.016)$. Therefore, cows expressing BoLA DRB3. $2 * 3$ and $* 11$ had the lowest SCC, whereas those 
Table 1. Means (SD), and numbers of cows with records for antibody-mediated immune responses (AMIR) and cell-mediated immune responses (CMIR), somatic cell counts, mastitis, and production data

\begin{tabular}{lcc}
\hline & No. of \\
Trait & cows & Mean (SD) \\
\hline AMIR, $^{1}$ ELISA optical density & 81 & $1.35(0.20)$ \\
CMIR antigen 1, \% increase, mm & 86 & $89(37)$ \\
CMIR antigen 2, \% increase, mm & 90 & $94(31)$ \\
LSCS, ${ }^{2}$ first lactation & 328 & $2.31(1.11)$ \\
LSCS, second lactation & 240 & $2.76(1.31)$ \\
LSCS, third lactation & 160 & $3.20(1.42)$ \\
Clinical mastitis, first lactation, \% of affected cows & 128 & $20.3(40.4)$ \\
Clinical mastitis, second lactation, \% of affected cows & 117 & $27.3(44.7)$ \\
Clinical mastitis, third lactation, \% of affected cows & 49 & $24.5(43.4)$ \\
305-d milk, first lactation, kg & 328 & $10,651(1697)$ \\
305-d protein, first lactation, kg & 328 & $337(47)$ \\
305-d fat, first lactation, kg & 328 & $398(57)$ \\
\hline
\end{tabular}

${ }^{1}$ ELISA optical density, d 21 postimmunization.

${ }^{2}$ Average percentage increase in skin thickness (mean of 24- and 48-h measurements).

${ }^{3} \mathrm{LSCS}=$ Lactation average of somatic cell scores.

expressing DRB3.2*23 had the highest SCC. Separate analyses by lactation also indicated that contrasts between allele $* 23$ and allele $* 11$, or between allele*23 and allele $* 3$ were significant $(P \leq 0.05)$ in the second and third lactations (data not shown).

Analyses restricted to the subset of cows for which information on the immune response was available showed less discrimination between BoLA DRB3.2 allele effects (Table 2). Nonetheless, in agreement with previous results on the complete data set, the contrast between allele $* 3$ and allele $* 23$ was significant $(P \leq$ $0.10)$ in the first and second lactation, respectively. When all first 3 lactations were considered simultaneously (repeatability model), the only significant results were the contrast between the effect of allele $* 3$ and the effect of allele $* 22(P=0.03)$. Therefore, cows expressing allele *3 had the favorable effect of a low SCS. The less

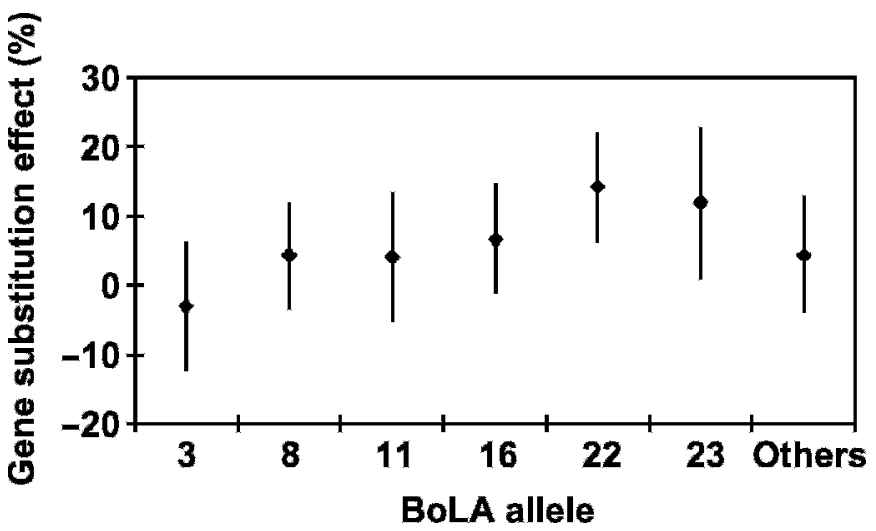

Figure 3. Bovine leukocyte antigen (BoLA) DRB3.2 allele substitution effect (SE) on average delayed-type hypersensitivity to antigen 2. No significant effect at $P \leq 0.05$ when compared with allele $* 24$. favorable effect of a higher SCS tended to be associated with alleles $* 22$ and $* 23$.

\section{Association Between BoLA DRB3.2 Alleles and Clinical Mastitis}

When compared with the reference allele BoLA DRB3.2*24, allele * 8 was associated with a significantly $(P \leq 0.05)$ higher risk of clinical mastitis (Figure 5). Moreover, the BoLA DRB3.2*3 allele associated with the lowest SCS was also associated with the lowest risk of clinical mastitis, which was significantly different $(P$ $\leq 0.05$ ) from the allele $* 8$ effect.

\section{Association Between BoLA DRB3.2 Alleles and Production Traits}

Associations between BoLA alleles and production traits were similar for milk, fat, and protein (Table 3).

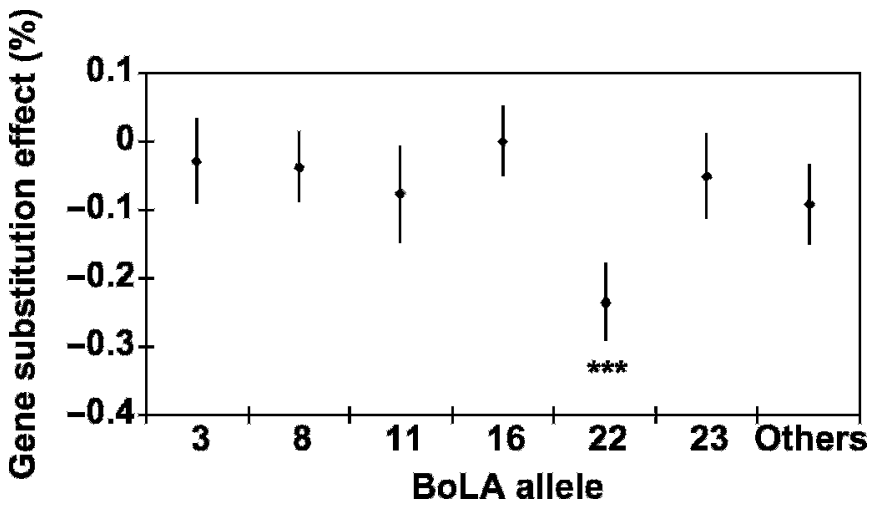

Figure 4. Bovine leukocyte antigen (BoLA) DRB3.2 allele substitution effect $( \pm \mathrm{SE}$ ) on $\mathrm{d} 21$ postimmunization with ovalbumin (antibodymediated immune response with ovalbumin). Significant effect at $P$ $\leq 0.001(* * *)$ when compared with allele $* 24$. 
Table 2. Bovine leukocyte antigen (BoLA) DRB3.2 allele substitution effect (estimate \pm SE) on lactation average of SCC (LSCS; repeated traits in first 3 lactations), when compared with allele $* 24^{1}$

\begin{tabular}{lcc}
\hline $\begin{array}{l}\text { BoLA DRB3.2 } \\
\text { allele }\end{array}$ & $\begin{array}{l}\text { LSCS, data set } 1^{2} \\
\text { (all animals) }\end{array}$ & $\begin{array}{l}\text { LSCS, data set } 2^{2} \\
\text { (animals with } \\
\text { immune response) }\end{array}$ \\
\hline$* 3$ & $-0.22(0.21)^{\mathrm{b}}$ & $-0.10(0.29)^{\mathrm{c}}$ \\
$* 8$ & $0.06(0.14)$ & $0.23(0.24)$ \\
$* 11$ & $-0.37(0.17)^{\mathrm{ab}}$ & $0.24(0.29)$ \\
$* 16$ & $0.07(0.16)$ & $0.30(0.25)$ \\
$* 22$ & $-0.06(0.16)$ & $0.41(0.29)^{\mathrm{c}}$ \\
$* 23$ & $0.31(0.20)^{\mathrm{b}}$ & $0.23(0.29)$ \\
Others & $0.17(0.14)$ & $0.12(0.24)$ \\
$* 24$ & $0.00^{\mathrm{a}}$ & 0.00 \\
\hline
\end{tabular}

${ }^{\mathrm{a}-\mathrm{C}}$ Superscript letters show different contrasts $(P \leq 0.05)$.

${ }^{1}$ Data set $1: \mathrm{n}=328$ cows and 831 lactations.

${ }^{2}$ Data set $2: \mathrm{n}=114$ cows with immune response information and 212 lactations.

Bovine leukocyte antigen DRB3.2*11 was significantly associated $(P \leq 0.05)$ with higher milk, fat, and protein yields when compared with the reference BoLA DRB3.2*24 allele, which exhibited nearly the lowest production yields. A favorable effect of allele*11 was also significant in analyses restricted to the subset of cows for which immune response data were available. Allele $* 23$ was associated with higher milk and protein production (Table 3 ), and associations with fat production also tended to be favorable $(P=0.10)$.

\section{Association Between BoLA DRB3.2 Alleles and Type Traits}

The effects of BoLA DRB3.2 alleles on type traits were not significantly different from the reference BoLA DRB3.2*24 allele. The only 2 significant contrasts $(P \leq$ 0.05 ) were unfavorable values (lowest) of type traits for cows carrying DRB3.2*16, when compared with cows

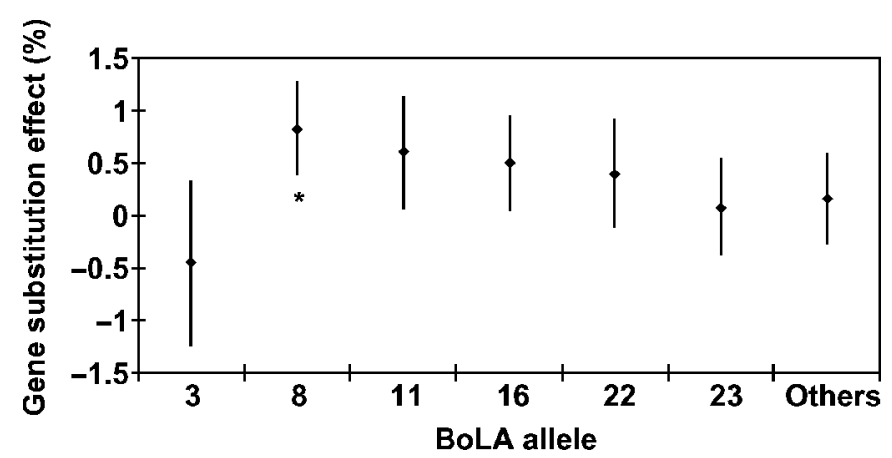

Figure 5. Bovine leukocyte antigen (BoLA) DRB3.2 allele substitution effect $( \pm \mathrm{SE})$ on clinical mastitis (repeated trait in first 3 lactations; $\mathrm{n}=128$ cows and 294 lactations). Significant effect at $P \leq 0.05$ $(*)$ when compared with allele *24.
Table 3. Bovine leukocyte antigen (BoLA) DRB3.2 allele substitution effect $( \pm \mathrm{SE})$ on milk, fat, and protein (repeated traits in the first 3 lactations), when compared with allele $* 24^{1}$

\begin{tabular}{lccc}
\hline $\begin{array}{l}\text { BoLA } \\
\text { DRB3.2 } \\
\text { allele }\end{array}$ & Milk, kg & Protein, kg & Fat, kg \\
\hline$* 3$ & $69(277)$ & $6.2(7,9)$ & $3.1(11.4)$ \\
$* 8$ & $-32(188)^{\mathrm{b}}$ & $2.0(5.1) \mathrm{d}$ & $2.2(6.5)$ \\
$* 11$ & $522(233)^{\mathrm{ab}}$ & $15.4(6.8)^{\mathrm{c}}$ & $18.8(8.0)^{\mathrm{ef}}$ \\
$* 16$ & $136(198)$ & $3.13(5.5)$ & $-0.4(7.3)^{\mathrm{f}}$ \\
$* 22$ & $260(202)$ & $6.2(5.6)$ & $9.3(7.0)$ \\
$* 23$ & $426(249)^{\mathrm{b}}$ & $17.6(7.5)^{\mathrm{cd}}$ & $16.5(9.3)$ \\
Others & $339(195)$ & $10.7(5.2)^{\mathrm{c}}$ & $1.9(6.3)$ \\
$* 24$ & $0^{\mathrm{a}}$ & $0^{\mathrm{c}}$ & $0^{\mathrm{e}}$ \\
\hline
\end{tabular}

${ }^{\mathrm{a}-\mathrm{f}}$ Superscript letters show different contrasts $(P \leq 0.05)$.

${ }^{1} \mathrm{n}=328$ cows and 831 lactations.

carrying DRB3.2*11, for capacity and to cows carrying DRB3.2*22 for udder depth.

\section{DISCUSSION}

The distribution of BoLA DRB3.2 allele frequencies was in good agreement with a previous large-scale survey on 835 Holstein cows in 56 Canadian herds (Sharif et al., 1998a). In both studies, alleles $* 8, * 22$, and $* 24$ had the highest frequencies $(\geq 12 \%)$, and the 7 most frequent alleles $(* 8, * 22, * 24, * 16, * 11, * 3$, and $* 23)$ represented 80 to $90 \%$ of allelic frequencies. Therefore, BoLA polymorphism information from the research herd seemed representative of the regional Holstein population. Moreover, average immune responses, SCC, and production traits were comparable to those reported elsewhere. Mean immune responses were in the range of previous reports of bovine AMIR (Wagter et al., 2000) and CMIR using similar test antigens (Hernández et al., 2005). Average LSCS ( \pm SD) in the first 3 lactations (Table 1) were comparable to values reported from large Canadian Holstein data sets (Boettcher et al., 1998), that is $2.18( \pm 1.22), 2.70( \pm 1.32)$, and 3.03 $( \pm 1.40)$ in the first, second, and third lactations, respectively. However, these results should be interpreted cautiously because of the limited size of the data, particularly for immune response traits.

In some cases, the results of associations between BoLA DRB3.2 and production traits were opposing, in that BoLA DRB3.2 alleles * 11 and $* 23$, which are associated with increased production traits, were associated with lower and higher LSCS, respectively. Moreover, the results were not consistent with previous results from Canadian cows (Sharif et al., 1998b) indicating favorable and unfavorable effects of alleles *8 and $* 22$, respectively. Therefore, it is difficult to draw any conclusions about a direct causal effect of BoLA alleles on production, but the findings lean toward a statistical 
linkage disequilibrium because of selection or spurious results. More generally, the multiplication of tests leads to increased type 1 error and false positive results.

Immune response was associated with BoLA DRB3.2 alleles $* 22, * 3$, and $* 24$. The literature widely indicates that polymorphism at the MHC loci contributes to quantitative differences in antibody production against a number of antigens in chickens (Lamont, 1991; Pinard-van der Laan, 2002), pigs (Mallard et al., 1989; Vaiman et al., 1998), goats (Eide et al., 1994), sheep (Garcia-Briones et al., 2000; Konnai et al., 2003), rats, mice, and humans (review by Puel and Mouton, 1996). However, the hierarchy of the response by various haplotypes and the range of their effects differed for each antigen, which Puel and Mouton (1996) related to the immunogenicity of the distinct epitopes. Therefore, use of test antigens other than those used in the present study to induce AMIR and CMIR may have led to different associations with other BoLA DRB3.2 alleles. However, selective breeding for high and low immune responses over several generations tends to reduce the effect of different antigens (Mallard et al., 1998). Moreover, the MHC complex comprises many genes that are in a strong linkage disequilibrium; therefore, a direct causal effect of BoLA DRB3.2 molecules in the present study cannot be disentangled from the effect of products of other linked genes.

One noteworthy result was that associations with BoLA DRB3.2 alleles were opposing for the 2 AMIR and CMIR traits, depending on the DTH antigen tested. Alleles $* 3$ and $* 24$ were associated with higher AMIR but lower DTH to Ag-2, whereas allele *22 was associated with lower AMIR but higher DTH to Ag-2. Such results place emphasis on the influence of the antigen, even though in this case the DTH test antigens were similar in nature. It is relevant to note that OVA and HEWL are type-2 antigens, whereas the antigens used to elicit DTH were type-1. These results also accentuate the pleiotropic role of BoLA molecules, or associated genes or gene products, on the cell- or antibody-mediated immune response. Depending on the AMIR and CMIR traits measured, it is not unusual for them to be in a reciprocal relationship, because these responses are controlled by type- 1 and type- 2 immune responses, respectively, and they tend to be cross-regulating (Rhodes and Graham, 2002; Raymond et al., 2006). Type-1 responses predominantly control intracellular pathogens and are dominated by IFN- $\gamma$, robust DTH, and the production of specific antibody isotypes, depending on the species (Sopp and Howard, 2001; Crawley et al., 2005). Conversely, type-2 responses predominately control extracellular pathogens and are dominated by IL-4, $-5,-10$, and -13 , and a robust antibody response (Rhodes and Graham, 2002). Moreover, this finding is in agreement with the hypothesis that both traits are genetically independent or opposite components of the immune response. Indeed, from their divergent selection for the antibody response in mice (Biozzi lines), Mouton et al. (1984) concluded that the expressions of AMIR and CMIR were at least partially under independent genetic regulation. Similarly, Pinard van der Laan (2002) conducted 3 divergent selection experiments in the chicken and concluded that selecting on only one criterion, antibody, leukocyte proliferation to mitogen, or phagocytic activity, did not necessarily modify other immunological traits.

Cows with low mean SCC over a given lactation (LSCS) are cows that exhibit the lowest inflammation of the mammary gland because of less severe or less durable IMI, or both. Such cows may be considered as the most resistant to mastitis. In this study, BoLA DRB3. $2 * 3$ and $* 11$ were associated with lower LSCS, whereas BoLA DRB3.2*22 and *23 were associated with higher LSCS. The favorable effect of BoLA DRB3.2*3 on LSCS was significant, whether complete ( $\mathrm{n}=328 \mathrm{cows}$ ) or reduced data sets including immune response information ( $\mathrm{n}=114$ cows $)$ were considered, when compared with $* 23$. However, the effect of allele *22 was not observed in the complete data set; therefore, all associations with allele $* 22$ in the reduced data set, including the associations with AMIR and CMIR, should be interpreted cautiously because they might have resulted from a statistical association by chance and not reflect any direct causal effect. Conversely, 2 associations between BoLA DRB3.2 alleles and LSCS were identified in the large data set (*11 compared with $* 24$, and *11 compared with *23) but not in the reduced data set, probably because the limited data size did not allow for detection of significant associations, especially for later lactations. Indeed, there were no differences in allele frequency distribution or in the average LSCS (and variability of LSCS) in the reduced or complete data sets. Previous studies also reported a favorable effect of BoLA DRB3.2*3 on SCC (Starkenburg et al., 1997) and a favorable effect of BoLA DRB3.2*11 on clinical mastitis in terms of less-reported cases (Kelm et al., 1997). However, Kelm et al. (1997) reported a contrasting unfavorable association between BoLA DRB3.2*3 and more IMI with minor pathogens. In agreement with the present results, the literature data indicated an unfavorable effect of BoLA DRB3.2*22 on SCC (Starkenburg et al., 1997), an unfavorable association of BoLA DRB3.2*23 with an increased risk of elevated SCC in third or greater lactations (Dietz et al., 1997), and an unfavorable association of BoLA DRB3.2*23 with an increased incidence of subclinical mastitis caused by bacterial pathogens (Ledwidge, 2003). In contrast to several previous reports, the pres- 
ent study did not identify an association between BoLA DRB3.2*16 and LSCS (Dietz et al., 1997; Kelm et al., 1997; Starkenburg et al., 1997; Sharif et al., 1998a). Also, allele DRB3.2*3 being related to lower LSCS was associated with less clinical mastitis in the current study, whereas allele BoLA DRB3.2*8 was associated with a higher mastitis risk. Unfavorable effects of allele *8 on mastitis have been reported by others (Dietz et al., 1997; Kelm et al., 1997, Sharif et al., 2000). Similarly, a favorable effect of allele $* 3$ was previously mentioned by Starkenburg et al. (1997). This allele was found to be associated with less acutely elevated SCC in the first lactation and showed a tendency toward an association with a lower risk of clinical mastitis. Results reporting that allele $* 24$ is associated with a lower risk of clinical mastitis are contradictory to literature results that show an unfavorable effect of this allele on clinical mastitis (Starkenburg et al., 1997; Sharif et al., 2000) and IMI with major pathogens (Kelm et al., 1997). These discrepancies have been notoriously difficult to avoid and are one of the strongest impediments to selective breeding based on the MHC genotype. This is not to say that information on MHC gene expression does not enhance the understanding of mechanisms involved in disease resistance (Alizadeh et al., 2003) or that it is not one of many tools that can be used to improve animal health in particular populations (Maillard et al., 2002, 2003).

Associations with BoLA alleles and LSCS, clinical mastitis, and immune response were partially correlated. Similar results for LSCS and clinical mastitis are in agreement with the high genetic correlation (0.70) between both traits (review by Rupp and Boichard, 2003), suggesting that these traits are partially determined by the same genes and common host defense mechanisms. It is also noteworthy that, in the present study, BoLA allele associations with immune responses were related to associations with mastitis resistance. For example, allele *3 was associated with increased antibody, as well as reduced mastitis and SCC. This could be due to an indirect relationship between the ability to produce antibodies and an enhanced defense against IMI caused by extracellular pathogens. Consequently, from our findings and from previous results (Starkenburg et al., 1997), the BoLA DRB3.2*3 allele may be a candidate for resistance to IMI, with the important caveat being its association with lower DTH to certain test antigens (e.g., Ag-2 in the current study).

Additionally, BoLA DRB3.2 alleles $* 22$ and $* 23$, which were associated with higher LSCS, share a similar AA motif in the binding groove with allele *8 (Sharif et al., 2000), which was found to be associated with higher clinical mastitis. These results, in addition to overwhelming data on the unfavorable associations of alleles $* 22, * 23$, and $* 8$ with udder health (Dietz et al., 1997; Starkenburg et al., 1997; Sharif et al., 1998a; Sharif et al., 2000) were in agreement with the finding of Sharif et al. (2000) that some AA motifs in the antigen-binding groove of BoLA DRB3.2*8, *22, and *23 are involved in susceptibility to mastitis. Also, allele $* 22$, which is unfavorable toward mastitis resistance, was found to be associated with decreased AMIR, again suggesting a link between AMIR and susceptibility to IMI. Collectively, these findings advocate the use of alleles $* 3, * 23$, and $* 22$ as reference points for more detailed mechanistic studies. It is also interesting to speculate on what keeps particular alleles at a higher frequency in this population of Canadian Holsteins, given that the alleles associated with the greatest production $(* 11, * 23)$, or the lowest risk of the most prevalent disease, mastitis $(* 3)$, were not those in the highest frequencies. In fact, these are the alleles of average frequency (Figure 1), which may suggest some benefit to moderating their influence.

\section{ACKNOWLEDGMENTS}

The authors gratefully acknowledge the technical assistance of Laura Wright at the University of Guelph, Dairy Research Station; Filippo Miglior of the Canadian Dairy Network for pedigree, production, and type trait information; and Korinne Hamilton in BoLA genotyping. The authors thank Bruce Wilkie for valuable comments. The research was supported by grants to Bonnie A. Mallard from the Natural Sciences and Engineering Research Council of Canada, the Ontario Ministry of Agriculture and Food, and DairyGen of Canada.

\section{REFERENCES}

Aarestrup, F. M., N. E. Jensen, and H. Østergård. 1995. Analysis of associations between major histocompatibility complex (BoLA) class I haplotypes and subclinical mastitis of dairy cows. J. Dairy Sci. 78:1684-1692.

Ali, A. K. A., and G. E. Shook. 1980. An optimum transformation for somatic cell concentration in milk. J. Dairy Sci. 63:487-490.

Alizadeh, Z., N. Karrow, and B. A. Mallard. 2003. Biological effect of varying peptide binding affinity to the BoLA-DRB3*2703 allele. Genet. Sel. Evol. 35(Suppl. 1):51-65.

Boettcher, P. J., J. C. M. Dekkers, and B. W. Kolstad. 1998. Development of an udder health index for sire selection based on somatic cell score, udder conformation, and milking speed. J. Dairy Sci. 81:1157-1168.

Crawley, A. M., B. A. Mallard, and B. N. Wilkie. 2005. Genetic selection for high and low immune response in pigs: Effects on immunoglobulin isotype expression. Vet. Immunol. Immunopathol. 108:71-76.

Dietz, A. B., N. D. Cohen, L. Timms, and E. J. R. Kehrli. 1997. Bovine lymphocytre antigen class II alleles as risk factors for high somatic cell counts in milk of lactating dairy cows. J. Dairy Sci. 80:406-412.

Eide, D. M., L. L. Nesse, and H. J. Larsen. 1994. Relation between the goat MHC (GLA) and antibody response to diphtheria toxoid, human serum albumin and tetanus toxoid, using a twin model. J. Anim. Genet. 111:234-242. 
Garcia-Briones, M. M., G. C. Russell, R. A. Oliver, C. Tami, O. Taboga, E. Carrillo, E. L. Palma, F. Sobrino, and E. J. Glass. 2000. Association of bovine DRB3 alleles with immune response to FMDV peptides and protection against viral challenge. Vaccine 19:1167-1171.

Hernández, A., N. Karrow, and B. A. Mallard. 2003. Evaluation of immune responses of cattle as a means to identify high or low responders and use of a human microarray to differentiate gene expression. Genet. Sel. Evol. 35(Suppl. 1):67-81.

Hernández, A., J. A. Yager, B.N. Wilkie, K. E. Leslie, and B. A. Mallard. 2005. Evaluation of bovine cutaneous delayed-type hypersensitivity (DTH) to various test antigens and a mitogen using several adjuvants. Vet Immunol Immunopathol. 104:45-58.

Kelm, S. C., J. C. Detilleux, A. E. Freeman, M. E. Jr. Kehrli, A. B. Dietz, L. K. Fox, J. E. Butler, I. Kasckovics, and D. H. Kelley. 1997. Genetic association between parameters of innate immunity and measures of mastitis in periparturient Holstein cattle. J. Dairy Sci. 80:1767-1775.

Konnai, S., S. N. Takeshima, S. Tajima, S. A. Yin, K. Okada, M. Onuma, and Y. Aida. 2003. The influence of ovine MHC class II DRB1 alleles on immune response in bovine leukemia virus infection. Microbiol. Immunol. 47:223-232.

Lamont, S. J. 1991. Immunogenetics and the major histocompatibility complex. Vet. Immunol. Immunopathol. 30:121-127.

Ledwidge, S. A. 2003. Associations between specific bovine leukocyte antigen DRB3 alleles and mastitis in Canadian Holsteins. MS Thesis. University of Guelph, Guelph, Ontario, Canada. NIH2101.

Maillard, J. C., I. Chantal, D. Berthier, S. Thevenon, I. Sidibe, and H. Razafindraibe. 2002. Molecular immunogenetics in susceptibility to bovine dermatophilosis: A candidate gene approach and a concrete field application. Ann. N. Y. Acad. Sci. 969:92-96.

Maillard, J. C., I. Chantal, D. Berthier, S. Thevenon, I. Sidibe, F. Stachurski, D. Belemsaga, H. Razafindraibe, and J. M. Elsen. 2003. Selection assisted by a BoLA-DR/DQ haplotype against susceptibility to bovine dermatophilosis. Genet. Sel. Evol. 35(Suppl 1):S193-S200.

Mallard, B. A., K. E. Leslie, J. C. M. Dekkers, R. Hedge, M. Bauman, and M. J. Stear. 1995. Differences in bovine lymphocyte antigen associations between immune responsiveness and risk of disease following intramammary infection with Staphylococcus aureus. J. Dairy Sci. 78:1937-1944.

Mallard, B. A., B. N. Wilkie, and B. W. Kennedy. 1989. The influence of the swine major histocompatibility genes (SLA) on variation in serum immunoglobulin (Ig) concentration. Vet. Immunol. Immunopathol. 21:139-145.

Mallard, B. A., B. N. Wilkie, B. W. Kennedy, J. Gibson, and M. Quinton. 1998. Immune responsivness in swine: Eight generations of selection for high and low immune response in Yorkshire pigs. Proc. 6th World Congress Genet. Appl. Livest. Prod. (Armidale, Australia) 27:257-262.

Mejdell, C. M., O. Lie, H. Solbu, E. F. Arnet, and R. L. Spooner. 1994. Association of major histocompatibility complex antigens (BoLAA) with AI bull progeny test results for mastitis, ketosis and fertility in Norwegian cattle. Anim. Genet. 25:99-104.

Mouton, D., Y. Bouthillier, J. C. Mevel, and G. Biozzi. 1984. Genetic selection for antibody responsivness in mice: Further evidence for inverse modification of macrophage catabolic activity without alteration of the expression of T-cell-mediated immunity. Ann. Immunol. 135:173-186.

Pinard-van der Laan, M. H. 2002. Immune modulation: The genetic approach. Vet. Immunol. Immunopathol. 87(3-4):199-205.
Puel, A., and D. Mouton. 1996. Genes responsible for quantitative regulation of antibody production. Crit. Rev. Immunol. 16:223250.

Raadsma, H. W., J. C. McEwan, M. J. Stear, and A. M. Crawford. 1999. Genetic characterisation of protective vaccine responses in sheep using multi-valent Dichelobacter nodosus vaccines. Vet. Immunol. Immunopathol. 72:219-229.

Raymond, C. R., A. M. Sidahmed, and B. N. Wilkie. 2006. Effects of antigen and recombinant porcine cytokines on pig dendritic cell cytokine expression in vitro. Vet. Immunol. Immunopathol. 111:175-185.

Rhodes, S. G., and S. P. Graham. 2002. Is "timing" important for cytokine polarization? Trends Immunol. 23:246-249.

Rothschild, M. F., L. Skow, and S. J. Lamont. 2000. Breeding for disease resistance in farm animals. Pages 73-105 in The Major Histocompatibility Complex and Its Role in Disease Resistance and Immune Responsiveness. R. F. E. Axford, S. C. Bishop, F. W. Nicholas, and J. B. Owen, ed. CAB International, Wallingford, Oxfordshire, UK

Rupp, R., and D. Boichard. 2003. Genetics of resistance to mastitis in dairy cattle. Vet. Res. 34:671-688.

Sharif, S., B. A. Mallard, and J. M. Sargeant. 2000. Presence of glutamine at position 74 of pocket 4 in the BoLA-DR antigen binding groove is associated with occurrence of clinical mastitis caused by Staphylococcus species. Vet. Immunol. Immunopathol. 76:231-238.

Sharif, S., B. A. Mallard, B. N. Wilkie, J. M. Sargeant, H. M. Scott, J. C. Dekkers, and K. E. Leslie. 1998a. Associations of the bovine major histocompatibility complex DRB3 (BoLA-DRB3) alleles with occurrence of disease and milk somatic cell score in Canadian dairy cattle. Anim. Genet. 29:185-193.

Sharif, S., B. A. Mallard, B. N. Wilkie, J. M. Sargeant, H. M. Scott, J. C. Dekkers, and K. E. Leslie. 1998b. Associations of the bovine major histocompatibility complex DRB3 (BoLA-DRB3) with production traits in Canadian dairy cattle. Anim. Genet. 30:157-160.

Sopp, P., and C. J. Howard. 2001. IFN $\gamma$ and IL-4 production by CD4, CD8 and WC1 $\gamma \delta \mathrm{TCR}^{+} \mathrm{T}$ cells from cattle lymph nodes and blood. Vet. Immunol. Immunopathol. 81:85-96.

Starkenburg, R. J., L. B. Hansen, M. E. Kehrli Jr, and H. ChesterJones. 1997. Frequencies and effects of alternative DRB3.2 alleles of bovine lymphocyte antigen for Holsteins milk selection and control lines. J. Dairy Sci. 80:3411-3419.

Stear, M. J., S. C. Bishop, B. A. Mallard, and H. Raadsma. 2001. The sustainability, feasibility and desirability of breeding livestock for disease resistance. Res. Vet. Sci. 71:1-7.

Vaiman, M., P. Chardon, and M. F. Rothschild. 1998. Porcine major histocompatibility complex. Rev. Sci. Tech. Off. Int. Epiz. 17:95-107.

van Eijk, M. J. T., J. A. Stewart-Haynes, and H. A. Lewin. 1992. Extensive polymorphism of the BoLA-DRB3 gene distinguished by PCR-RFLP. Anim. Genet. 23:483-496.

Wagter, L. C., B. A. Mallard, B. N. Wilkie, K. E. Leslie, P. J. Boettcher, and J. C. M. Dekkers. 2000. A quantitative approach to classifying Holstein cows based on antibody responsiveness and its relationship to peripartum mastitis occurrence. J. Dairy Sci. 83:488-498.

Weigel, K. A., A. E. Freeman, M. E. Kehrli, M. J. Stear, and D. H. Kelley. 1990. Association of class I bovine lymphocyte antigen complex alleles with health and production traits in dairy cattle. J. Dairy Sci. 73:2538-2546.

Xu, A., M. J. Van Eijk, C. Park, and H. A. Lewin. 1993. Polymorphism in BoLA-DRB3 exon 2 correlates with resistance to persistent lymphocytosis caused by bovine leukemia virus. J. Immunol. 151:6977-6985. 